\title{
THEORETICAL METHOD FOR PREDICTING THE PROPERTIES OF CYCLOTRON HARMONIC WAVES FROM THE PERPENDICULAR DISPERSION RELATION
}

\section{B. Lembege.}

Space Plasma Physics Division Space Science Department, European Space Agency, Noordwijk, The Netherlands

A theoretical method is proposed for predicting the properties of the backward propagating cyclotron harmonic waves (CHV) from the simple dispersion curve for perpendicular propagation. This method is illustrated for the frequency range $1<\omega / \omega_{c}<2$, where $\omega$ and $\omega_{c}$ are respectively the wave and electron cyclotron frequencies and is applicable for any plasma density conditions.

In previous experimental and theoretical study (Lembège, 1979), it was proposed a new classification of properties of CHW both in the propagation and detection plane respectively described by the normalized wave vector $\overrightarrow{k p}$ and the distance vector $\vec{r} ; 0$ is the electron gyroradius. This classification is based on the determination of two groups of values $\omega / \omega_{c}$ separated by a certain boundary value ( $\left.\omega_{\mathrm{Cy}} /{ }^{\prime}{ }_{\mathrm{C}}\right)_{1}$. Knowing a given high value of $\left(\omega_{p} / \omega_{c}\right)^{2}$, where $\omega_{p}$ is the plasma frequency, the dispersion curve for perpendicular propagation can be numerically determined (Stix, 1962) ; consequently the value $\left(\omega / \omega_{c}\right) p_{1}$ of its inflexion point $P_{1}$ can be easily known. Then it was shown that the value ( $\left.\omega_{\text {cyl }} / \omega_{c}\right)_{1}$ can be quickly defined from $P_{1}$ by the numerical relation $\left(\omega_{\text {cyl }} / \omega_{C}\right)_{1}=\left(\omega / \omega_{C}\right) p_{1}-\delta_{1}$, where $\delta_{1}$ is roughly constant for dense plasma conditions and equal to 0.145 . This point ${ }^{P}$ cyl, l was shown to present particular characteristics and to divide the frequency range $I<\omega / \omega_{c}<2$ into two groups $I$ and II. However, it can be shown that although the classification into two groups I and II is always applicable for any plasma conditions, the previous principle of determination of the boundary value $\left(\omega_{\mathrm{cyl}} / \omega_{\mathrm{c}}\right)_{l}$ cannot be simply extended to

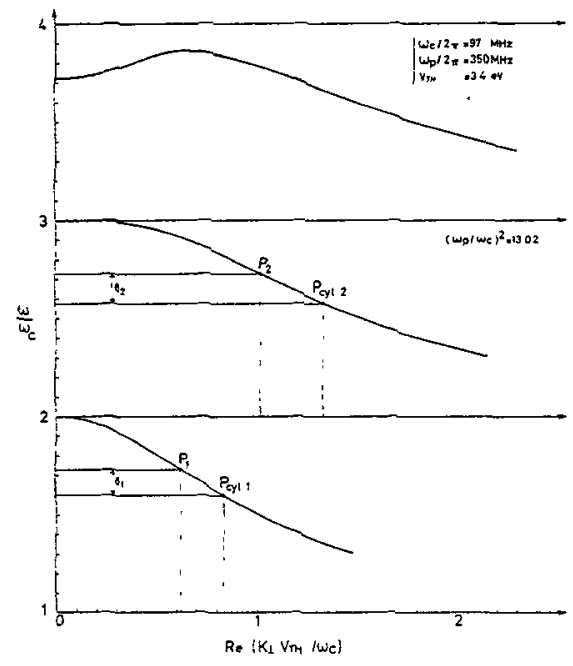

Figure 1: Theoretical curves of the dispersion relation in the perpendicular propagation for on example of dense plasma $\left(\omega_{p} / \omega_{c}\right)^{2}=13.02$; the indexes 1 and 2 of $P$ and $P_{\text {cy }}$ refer to the dispersion bronches of order 1 and 2 .

other plasma densities. This is due to the big variation of $\delta_{l}$ with $\left(\omega_{p} / \omega_{c}\right)^{2}$ for mean and low plasma densities (Figure 2).

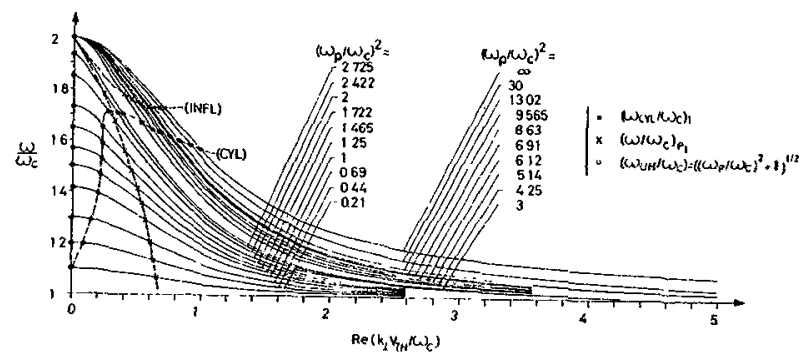

Figure 2 : Theoretical curves of the first dispersion branch in the perpendicular propagation for $\left(\omega_{p} / \omega_{c}\right)^{2}$ varying from infinity to very zow values. The curve (CYL) (.-....) joins the different values of $\left(\omega_{c y} / \omega_{c}\right)_{1}$. The curve (INiL) (-) joins the locations of the various inflexion points $P_{1}$. 
Presently, the locations of $\mathrm{P}_{\text {cyl, }}$ are determined for a large number of values of $\left(\omega_{\mathrm{p}} / \omega_{\mathrm{C}}\right)^{2}$ from very low to infinite plasma densities (Figure 2) ; each point $P_{C y l, 1}$ is numerically defined by the frequency $\omega / \omega_{c}$ for which the low damped part AI of the polar curves $k \rho$ has almost zero curvature with respect to the origin $O$ (Figure 3 ); in this case $A I=A_{C y l} I_{C y l}$.

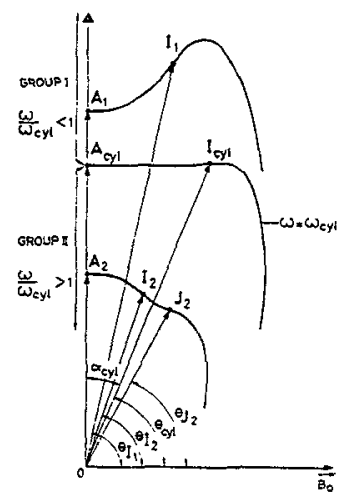

Figure 3 : Sketch of the three kinds of polar curves of the real part of $k p$ inside the frequency range $1<\omega / \omega_{c}<2$ (not to scale).

The ensemble of the points $P_{c y l, l}$ determines a curve (CYL) which is used as a reference curve; this curve divides the plane $\left(\omega / \omega_{\mathrm{C}} ; \operatorname{Re}\left(\mathrm{k}_{\perp} \mathrm{v}_{\mathrm{TH}} / \omega_{\mathrm{C}}\right)\right)$ into two areas $I$ and II characteristic of the two groups previously defined (Figure 4).

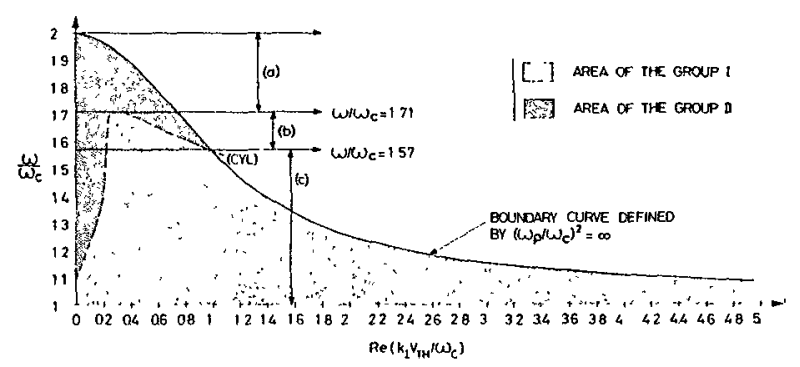

Figure 4 : Representation of the three characteristic frequency ronges $(a),(b)$ and $(c)$ of $\omega / \omega_{c}$ respectively defined by $(2,1.71),(1.71,1.57)$ and $(1.57,1)$.
The present method consists of varying the ratio $\omega / \omega_{c}$ represented by a straight line (L) parallel to the axis $\operatorname{Re}\left(k_{\perp} V_{T H} / \omega_{C}\right)$ and noting where the line (L) intersects the curve (CYL). Three different frequencies ranges of $\omega / \omega_{c^{\prime}}(a),(b)$ and (c) can be defined. It is shown that as a function of the ratio $\omega / \omega_{c}$, the number of intersections between ( $L$ ) and (CXL), and various ranges of $\left(\omega_{p} / \omega_{c}\right)^{2}$ can be also defined $;$ in each one of these ranges, the properties of CHW can be easily obtained using the characteristics of the groups $I$ and II. This method is shown to be extended to other dispersion branches of backward propagating $\mathrm{CHW}$ for perpendicular propagation.

\section{References : - Lembège (1979), Antenna} radiation pattern of cyclotron harmonic waves in a hot magnetoplasma, Rad. Science (to be published in May-June).

$$
\text { - Stix (1962), Theory of }
$$

Plasma waves, Mc Graw Hill, New York. 